differs from zero only on a denumerable set of $\xi$ 's.

\title{
REFERENCES
}

1. Nelson Dunford, On one-parameter groups of linear transformations, Ann. of Math. vol. 39 (1938) pp. 569-573.

2. Einar Hille, Functional analysis and semi-groups, Amer. Math. Soc. Colloquium Publications, vol. 31, New York, 1948.

3. Emile Picard, Leģons sur quelques equations fonctionnelles, Paris, 1928, p. 3.

The University of Southern California

\section{MEAN VALUES AND FRULLANI INTEGRALS}

\section{RALPH PALMER AGNEW}

1. Introduction. Let $I(a, b)$ denote the Frullani integral defined by

$$
\begin{aligned}
I(a, b) & =\int_{0}^{\infty} \frac{f(a t)-f(b t)}{t} d t \\
& =\lim _{\epsilon \rightarrow 0, h \rightarrow \infty} \int_{\epsilon}^{h} \frac{f(a t)-f(b t)}{t} d t \\
& =\lim _{\epsilon \rightarrow 0, h \rightarrow \infty}\left[\int_{a \epsilon}^{a_{h}} \frac{f(t)}{t} d t-\int_{b \epsilon}^{b h} \frac{f(t)}{t} d t\right] \\
& =\lim _{h \rightarrow \infty} \int_{b h}^{a_{h}} \frac{f(t)}{t} d t-\lim _{\epsilon \rightarrow 0} \int_{b \epsilon}^{a_{\epsilon}} \frac{f(t)}{t} d t
\end{aligned}
$$

when the limits exist; it is assumed that $a, b>0$ and $f(t)$ is Lebesgue integrable over each interval $0<m \leqq t \leqq M<\infty$. If in the last two integrals we put $t=e^{u}$ and $t=e^{-u}$ respectively, and set

$$
\lambda=\log (a / b)
$$

to simplify formulas, we find that

$$
I(a, b)=\lim _{A \rightarrow \infty} \int_{A}^{\lambda+A} f\left(e^{u}\right) d u+\lim _{B \rightarrow \infty} \int_{B}^{-\lambda+B} f\left(e^{-u}\right) d u
$$

when the limits exist. From (3) we obtain immediately the Frullani formula

$$
I(a, b)=\lambda\left[\lim _{x \rightarrow \infty} f(x)-\lim _{x \rightarrow 0} f(x)\right]
$$

whenever these limits exist.

Presented to the Society, April 29, 1950; received by the editors April 10, 1950. 
Iyengar [1940] and Agnew [1942] have studied the question of existence of $I(a, b)$ by studying

$$
\lim _{\Lambda \rightarrow \infty} \int_{\Lambda}^{\lambda+\Lambda} f\left(e^{u}\right) d u .
$$

They showed that if the limit in (5) exists for each $\lambda$ in a set having positive measure, then it exists for each real $\lambda$ and has the value $\lambda L$ where $L$ is a constant and, moreover,

$$
\lim _{\Lambda \rightarrow \infty} e^{A} \int_{A}^{\infty} f\left(e^{u}\right) e^{-u} d u=L .
$$

On the other hand if (6) holds, then the limit in (5) exists and is $\lambda L$ for each real $\lambda$. Although neither Iyengar nor Agnew wrote the formula, this implies that if $I(a, b)$ exists for each $\lambda$ in a set of positive measure, then (6) and, for some constant $L^{\prime}$,

$$
\lim _{B \rightarrow \infty} e^{B} \int_{B}^{\infty} f\left(e^{-u}\right) e^{-u} d u=L^{\prime}
$$

hold and

$$
I(a, b)=\lambda\left[L-L^{\prime}\right] .
$$

On the other hand, if the limits in (6) and (7) exist, then (8) holds for each pair of positive numbers $a$ and $b$.

In a recent paper, Ostrowski [1949] has given the formula (8) with $L$ and $L^{\prime}$ defined by

$$
L=\lim _{x \rightarrow \infty} \frac{1}{x} \int_{0}^{x} f(t) d t ; \quad L^{\prime}=\lim _{x \rightarrow 0} x \int_{x}^{1} \frac{f(t)}{t^{2}} d t .
$$

The similarity of this result with that given above has caused the author to examine (6) and (7). If in (6) and (7) we put $t=e^{u}$ and $t=e^{-u}$ respectively we get

$$
L=\lim _{x \rightarrow \infty} x \int_{x}^{\infty} \frac{f(t)}{t^{2}} d t ; \quad L^{\prime}=\lim _{x \rightarrow 0} \frac{1}{x} \int_{0}^{x} f(t) d t .
$$

2. Two theorems on mean values. The results given above imply the two following theorems on mean values; in each case

$$
F(a, b)=\int_{a}^{b} \phi(t) d t
$$

is a Lebesgue integral when $0<a<b<\infty, F(0, b)$ is defined as 
$\lim _{a \rightarrow 0} F(a, b)$, and $F(a, \infty)$ is defined as $\lim _{b \rightarrow \infty} F(a, b)$.

THEOREм 1. If $f(x)$ is Lebesgue integrable over each finite interval $0<a \leqq x \leqq b<\infty$ and one of the mean values in

$$
\lim _{x \rightarrow \infty} \frac{1}{x} \int_{1}^{x} f(t) d t=\lim _{x \rightarrow \infty} x \int_{x}^{\infty} \frac{f(t)}{t^{2}} d t
$$

exists, then both exist and the equality holds.

THEOREM 2. If $f(x)$ is Lebesgue integrable over each finite interval $0<m<x<M<\infty$ and one of the mean values in

$$
\lim _{x \rightarrow 0} \frac{1}{x} \int_{0}^{x} f(t) d t=\lim _{x \rightarrow 0} x \int_{x}^{1} \frac{f(t)}{t^{2}} d t
$$

exists, then both exist and the equality holds.

Because simple straightforward proofs of these theorems can be given, it seems proper to give them here because the proofs outlined above involve much irrelevant and difficult material some of which is not yet published; Ostrowski [1949] says proof of his result involving the limits in (9) "is difficult and will be given in another publication." To prove Theorem 1 , which involves mean values at $\infty$, suppose first that the left member of (11) exists and has the value $L$. Let

$$
F(x)=\frac{1}{x} \int_{1}^{x} f(t) d t
$$

so that $F(x) \rightarrow L$ as $x \rightarrow \infty$. Integration by parts gives

$$
\left.x \int_{x}^{\infty} \frac{f(t)}{t^{2}} d t=\frac{x}{t} F(t)\right]_{x}^{\infty}+2 x \int_{x}^{\infty} \frac{F(t)}{t^{2}} d t .
$$

Since $t^{-1} F(t) \rightarrow 0$ as $t \rightarrow \infty$, the first term on the right has the value $-F(x)$ and this converges to $-L$ as $x \rightarrow \infty$. It is easy to show directly (or by application of the theory of kernel transformations of functions given in Hardy [1949], p. 50) that the second term converges to $2 L$ as $x \rightarrow \infty$. Thus the right member of (11) exists and has the value $L$. Suppose now that the right member of (11) exists and has the value L. Let

$$
G(x)=x \int_{x}^{\infty} \frac{f(t)}{t^{2}} d t
$$

so that $G(x) \rightarrow L$ as $x \rightarrow \infty$. Integration by parts gives 


$$
\begin{aligned}
\frac{1}{x} \int_{1}^{x} f(t) d t & =\frac{1}{x} \int_{1}^{x} \frac{f(t)}{t^{2}} t^{2} d t \\
& \left.=-\frac{t}{x} G(t)\right]_{1}^{x}+\frac{2}{x} \int_{1}^{x} G(t) d t .
\end{aligned}
$$

The first term of the last member is $-G(x)+x^{-1} G(1)$ and this converges to $-L$ as $x \rightarrow \infty$; and the last term converges to $2 L$ as $x \rightarrow \infty$. Thus the left member of (11) exists and has the value $L$. This completes the proof of Theorem 1 .

Theorem 2 may be proved by similar integrations by parts, or more satisfyingly by changes of variable (put $t=1 / u$ ) and notation which show that Theorem 2 is a consequence of Theorem 1. In fact

$$
\lim _{x \rightarrow 0} \frac{1}{x} \int_{0}^{x} f(t) d t=\lim _{x \rightarrow 0} \frac{1}{x} \int_{1 / x}^{\infty} \frac{f\left(u^{-1}\right)}{u^{2}} d u=\lim _{x \rightarrow \infty} x \int_{x}^{\infty} \frac{f\left(t^{-1}\right)}{t^{2}} d t
$$

whenever one of the limits exists.

3. Integrals of differences of translations. By use of Theorems 1 and 2 we are able to put conclusions of the author [1942] concerning the integral

$$
I(\lambda)=\int_{-\infty}^{\infty}[g(t+\lambda)-g(t)] d t
$$

in a much more satisfactory form. The function $g(t)$ is assumed to be Lebesgue integrable over each finite interval of values of $\lambda$, and $I(\lambda)$ is defined by

$$
I(\lambda)=\lim _{A \rightarrow-\infty, B \rightarrow \infty} \int_{A}^{B}[g(t+\lambda)-g(t)] d t
$$

whenever the limit exists. Our improved theorem is the following.

THEOREM 3. If $I(\lambda)$ exists for each $\lambda$ in a set having positive measure, then the ordinary mean values

(20) $M=\lim _{x \rightarrow \infty} \frac{1}{x} \int_{1}^{x} g(\log u) d u, \quad M^{\prime}=\lim _{x \rightarrow \infty} \frac{1}{x} \int_{-x}^{-1} g\left(-\log |u|^{-}\right) d u$

of $g(\log u)$ and $g(-\log |u|)$ at $u=\infty$ and $u=-\infty$, respectively, both exist and the formula

$$
I(\lambda)=\lambda\left[M-M^{\prime}\right]
$$

holds for each real $\lambda$. Conversely, if the mean values $M$ and $M^{\prime}$ exist 
then $I(\lambda)$ exists and (21) holds for each real $\lambda$.

4. Frullani integrals. Using the results of Iyengar and Agnew given above, or, alternatively, those of Ostrowski, and Theorems 1 and 2, we obtain the following incisive theorem involving Frullani integrals and the familiar mean values of $f(x)$ at 0 and at $\infty$.

THEOREм 4. Let $f(t)$ be Lebesgue integrable over each finite interval $0<m \leqq t \leqq M<\infty$. If the Frullani integral in the left member of

$$
\int_{0}^{\infty} \frac{f(a t)-f(b t)}{t} d t=\lambda\left[\lim _{x \rightarrow \infty} \frac{1}{x} \int_{0}^{x} f(t) d t-\lim _{x \rightarrow 0} \frac{1}{x} \int_{0}^{x} f(t) d t\right]
$$

exists for each $\lambda=\log (a / b)$ in a set of positive measure, then the mean values in the right member exist and (22) holds for each pair of positive numbers $a$ and $b$. On the other hand, if the mean values in the right member of (22) exist, then the left member exists and the formula holds for each pair of positive numbers $a$ and $b$.

\section{REFERENCES}

R. P. Agnew, Limits of integrals, Duke Math. J. vol. 9 (1942) pp. 10-19.

G. H. Hardy, Divergent series, Oxford, 1949, 396 pp.

K. S. K. Iyengar, On Frullani integrals, Journal of the Indian Mathematical Society (2) vol. 4 (1940) pp. 145-150; reprinted, Proc. Cambridge Philos. Soc. vol. 37 (1941) pp. 9-13.

A. M. Ostrowski, On some generalizations of the Cauchy-Frullani integral, Proc. Nat. Acad. Sci. U.S.A. vol. 35 (1949) pp. 612-616.

Cornell University 